\title{
Gender Prediction by Face Features Extraction and Fuzzy Rules
}

\author{
Surinder Kaur \\ Computer Science \& Engg, GGITS, JBP
}

\author{
Preeti Rai \\ Computer Science \& Engg,GGITS, JBP
}

\begin{abstract}
Over the current years, a phenomenal arrangement of exertion has been made to sex forecast from confront pictures. it's been supposed that age will be precisely measurable beneath controlled air comparable to frontal faces, no demeanor, and static lighting conditions. Be that as it may, it is difficult to accomplish an identical exactness level in true climate because of broad varieties secretly settings, facial postures, and brightening conditions. amid this paper, we have a tendency to apply an as of late proposed machine learning strategy alluded to as covariate move adjustment to alleviating lighting condition alteration amongst research center and sensible environment. Through certifiable age estimation tests, we have a tendency to exhibit the utility of our anticipated strategy.
\end{abstract}

\section{Keywords}

Face Detection, Skin Color Segmentation, Face Features extraction, Features recognization, Fuzzy rules.

\section{INTRODUCTION}

Human's countenances uncover various information and additionally sex, age and quality. they supply essential signals for a few applications, tantamount to distinguishing proof and smart human-PC interface. amid this paper, we tend to blessing a substitution method which will build up people's sexual orientations from their face pictures. inside the previous, a few inquires about give to discovering savvy picture choices for sex acknowledgment. Among them, Adaboost [1]may be a brilliant instrument for highlight decision.

Shakhnarovich and Viola [6] utilized Viola and Jones' fell AdaBoost procedure to mentor a face finder, that might be a straight blend of the powerless classifiers assigned from parallelogram alternatives. They at that point assigned an accumulation of powerless classifiers from the face indicator for sexual orientation and quality acknowledgment.

Among the on top of, the Shakhnarovich and Viola's procedure [6] is maybe the preeminent conservative one regarding the machine an incentive for genuine applications. It's because of that some of the parallelogram choices utilized for confront identification ar re-utilized for sexual orientation acknowledgment. Consequently, amid this method, we have a tendency to don't should recomputed the choices once the face has been identified, so the entire machine time (counting each face discovery and sex acknowledgment) will be decreased. Moreover, it's conjointly recorded that the examination of parallelogram choices will be altogether quickened by exploitation the basic picture.

As opposed to parallelogram choices, Shummet and Henry [7] planned a substitution set of feeble classifiers that utilization the association between 2 pixels' forces as alternatives, and demonstrate that the notoriety rate will be extra made strides.
As opposed to AdaBoost, Moghaddam and control [8] explored the use of nonlinear help vector machines (SVMs) to order sexual orientation from confront pictures. With the mathematician spiral premise perform $(\mathrm{RBF})$ piece, a high. Be that as it may, the machine various these 2 approaches are high. They're so not suitable for period applications system.

\section{LITERATURE SURVEY}

Recognizing the age and sexual orientation information of a speaker given a concise discourse sound-related correspondence could be a troublesome assignment and has increased essential consideration as of late. Metze et al. [1] thought about four methodologies for age and sex acknowledgment from telephone discourse; these encased a parallel discourse sound recognizer framework to check the Viterbi mystery composing scores for each class particular discourse sound recognizer, a framework abuse dynamic hypothesis systems to blend numerous discourse alternatives, a framework essentially construct solely with respect to straight expectation investigation, and a GMM framework upheld MFCCs. it had been supposed in Metze et al. [1] that the parallel telephone recognizer framework performs in like manner as human audience members on long articulations however its execution debases on short expressions while the framework upheld discourse choices, reminiscent of key (F0), jitter, gleam and music to-clamor proportion, has demonstrated relative healthiness to the variety of the soundrelated correspondence length.

In Ajmera and Burkhardt[2], the distinctive cos update is associated with the cepstral coefficients and besides the cepstral bearings much the same as lower $(3-14 \mathrm{~Hz})$ change frequencies offer best division. Talk decisions (pitch, essentialness, formants, vocal tract length twisting issue, talking rate, et cetera) and their important can even be intercalary to the cepstral options at the edge or sound-related correspondence level to help the execution besides to the talk decisions, novel lexical level decisions like word-class frequencies have additionally been foreseen for age affirmation reason.

(Wolters et al.). Inside the fluffy SVM demonstrating system anticipated by Nguyen[3]. A fluffy enrollment is appointed as a weight to each training datum to expand the robustness against commotion and exceptions. In addition, methods from speaker confirmation and dialect distinguishing proof applications reminiscent of GMM-SVM mean super vector frameworks, aggravation quality projection (NAP).

In Dobry et al.[3], grapple displaying uses a posterior SVM to demonstrate the circulation of closeness scores between instructing learning and each one the stay speaker models. in view of the different parts of displaying, consolidating totally unique characterization methodologies along will for the most part extensively enhance the execution.

People comprehend sexual orientation not exclusively upheld 
the face, however furthermore on the surrounding setting reminiscent of hair, covering and skin tone [6], stride and furthermore the entire body. Underneath, we tend to survey applicable work on sex expectation from facial pictures exclusively. the matter of sexual orientation order bolstered human appearances has been widely contemplated inside the writing. There are 2 in vogue methodologies. the essential one is anticipated by Moghaddam et al. [7] wherever a Support Vector Machine (SVM) is utilized for sexual orientation characterization bolstered fingernail confront pictures. The second was given by Baluja .World Health Organization connected the Adaboost algorithmic program for sexual orientation expectation. As of late, on account of the acknowledgment of local Binary Patterns (LBP) in confront acknowledgment applications, Yang utilized LBP bar graph choices for sexual orientation include delineation, and furthermore the Adaboost algorithmic program to be told the best local choices for arrangement. Examinations were performed to foresee age, sexual orientation and quality from confront pictures. a similar approach was anticipated in [8]. elective local descriptors have furthermore been received for sexual orientation arrangement. Wang et al. [9] anticipated a totally one of a kind sexual orientation acknowledgment strategy abuse Scale Invariant Feature redesign (SIFT) descriptors and frame settings. By and by, Adaboost was wont to pick alternatives from confront pictures and sort a strong classifier.Gao et al. [10] performed confront construct sexual orientation arrangement with respect to customer pictures no heritable from a multi-ethnic face data. to beat the nonconsistency of make, expression, and light changes, they anticipated the use of Active shape Models (ASM) to standardize facial surface. The work finished that the prospect of ethnic variables will encourage enhance sex characterization exactness amid a multi-ethnic setting. a logical synopsis regarding the matter of sexual orientation grouping from confront pictures are regularly found in [11].

Among every one of the descriptors that write in code sexual orientation information reminiscent of LBP [12].The LBP has demonstrated brilliant segregation ability while keeping up effortlessness [13]. to determine a benchmark for look essentially based procedures, we tend to utilize LBP together with SVM to anticipate sexual orientation from facial pictures amid this work. in spite of the fact that in past work [14] unadulterated arithmetic alternatives were utilized as from the earlier information to help enhance grouping execution, none of the aforementioned approaches, as opposed to our work, directed explicitly and only on facial science as a path for sexual orientation order. possibly our work is a great deal of nearly identifying with prior investigation by Shi et al. [15] on confront acknowledgment abuse geometric choices, wherever they utilized quantitative connection choices registered from numerous anatomical points of interest. Be that as it may, we tend to take a great deal of thorough look at the express utilization of facial unadulterated science in assurance the matter of sexual orientation grouping. we tend to utilize only science information upheld points of interest, which can or won't not be naturally intentional. In our approach, the local information from independent points of interest is utilized as opposed to all encompassing information from all milestones.

\section{PROPOSED ALGORITHM 3.1 Gender Recognization}

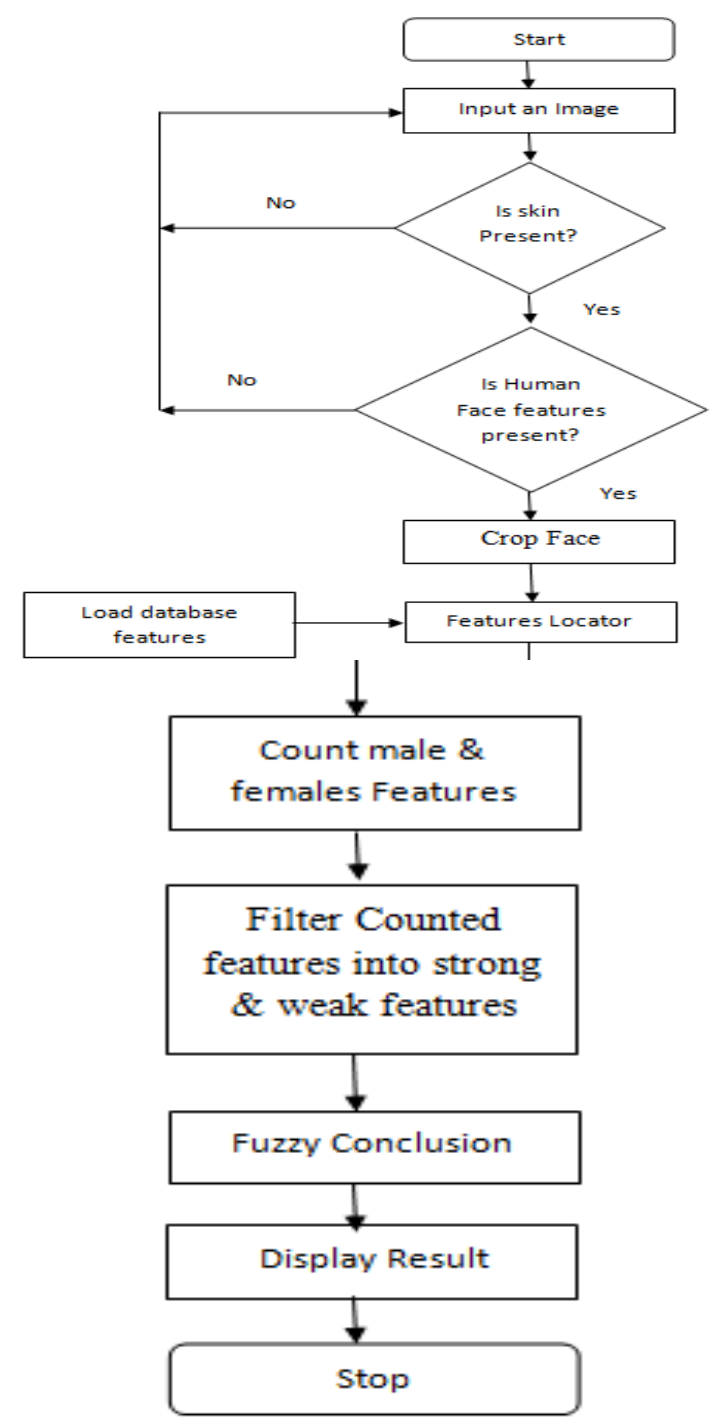

Figure 3.1 Data Flow diagram of Gender Recognization

1. Input a picture

2. Discover skin space in Input Image.

3. Discover options like eyes and mouth, nose in skin region.

4. If options detected then move to step five else step one.

5. Crop Face.

6. Load information Male Females options.

7. Find options in an exceedingly face space.

8. Count Male \&amp; feminine options.

9. Filter Counted options into robust \&amp; weak options.

10. Kind Fuzzy Conclusion from options \&amp; show gender result.

\subsection{Face Detection}

Skin color plays a vital role in differentiating human and nonhuman faces. From the study it is observe that skin color pixels have a decimal value in the range of 120 to 180. In this 
project, we used a trial and error method to locate skin color and non skin color pixels. But many of the times, system fails to detect whether an image contains human face or not (i.e. for those images where there is a skin color background).an image is segmented into skin color and non-skin color pixels with the equations

$$
\begin{array}{r}
120 \leq \mid \text { Pxy } \mid \leq 180 \ldots \ldots \ldots \text {.eq } 3.1 \\
\text { where } P x y=\text { pixel at position } x y
\end{array}
$$

The skin pixels values are set to 1(i.e. \#FFFF) and non skin pixels are set to 0(i.e. 0000). The pixels are collected and set as per equation

If

$\lim _{i \rightarrow 1} n\left(\int_{1}^{3} 120 \leq|P x y| \leq 180\right)=1$

Else

$\lim _{i \rightarrow 1} n\left(\int_{1}^{3} 180 \leq|P x y| \leq 120\right)=0$

where $n=$ total number of pixels of input image

Writing audit call attention to that, FACS framework strategy depends on confront highlights extractions like eye, nose, mouth, and so on. In this venture, we limit the quantity of components (i.e. just eyes and mouth) yet given the more weight age for fluffy standards arrangements from these extricated highlights. Face extractions comprise of following strides.

- Let $\mathrm{W}$ and $\mathrm{H}$ are the width and stature of skin and non-pixel picture as appeared in fig 3.1.1

- $\quad$ Read the pixel at position $(0, \mathrm{H} / 2)$ which is a center of i.e. left half of picture.

- $\quad$ Travers a separation D1 = W/6 in flat heading to get the begin limit pixel of skin district.

- Travers a separation $\mathrm{D} 2=\mathrm{H} / 6$ from a pixel position (W/6, H/2) in upward bearings. Same may do descending way and find the focuses $\mathrm{X} 1, \mathrm{X} 2$.

- Travers a separation D3=W/3 from the point X1 and find the point $\mathrm{X} 3$. Same do from the point $\mathrm{x} 2$ and find the point $\mathrm{X} 4$.

- $\quad$ Crop the square picture as appeared.

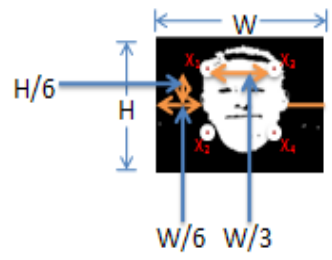

Figure 3.2 Detected Face Area

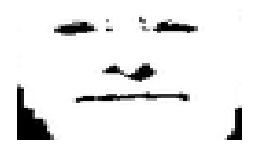

Figure 3.3 Face Features Extraction

Human face is comprised of eyes; nose, mouth and chine and so on there are contrasts fit as a fiddle, size, and structure of these organs. So the countenances are contrasts in thousands way. One of the regular techniques for confront demeanor acknowledgment is to remove the state of eyes and mouth and after that recognize the appearances by the separation and size of these organs. The face include extractions comprise of following strides.

- Let $\mathrm{W}$ and $\mathrm{H}$ are width and tallness of a picture appeared in Fig 3.2.3

- Mark pixel Pi (W/2, H/2) as focal point of picture.

- Travers a separation H/8 from the pixel Pi towards upward and stamp a point $\mathrm{K} 1$.

- Travers a separation W/3 from the point K1 towards leftward and stamp a point K2.

- Travers a separation $\mathrm{H} / 10$ towards descending from the point $\mathrm{K} 2$ and stamp a point $\mathrm{K} 3$.

- Travers a separation W/4 from the point K3 towards right and stamp the point $\mathrm{K} 4$.

- Travers a division $\mathrm{H} / 10$ from the direct $\mathrm{K} 4$ to up and check the point $\mathrm{K} 5$.

- $\quad$ Same steps are reiterated for isolating the right eye and stamp the point N2, N3, N4, and N5.

- Travers a separation H/8 from the point Pi towards descending and check the point M1.

- Travers a separation W/6 towards left and ideal from the point M1 and imprints the point M2 and M3.

- Start with the point M2 navigate a separation H/10 towards descending and check the point M4.

- Travers a separation W/6 from the point M4 towards right and check the point M5. Same may do from point M5 and stamp the point M6.

- Travers the separation H/10 from M6 towards up that meets to the point M3.

- $\quad$ See the beneath picture.

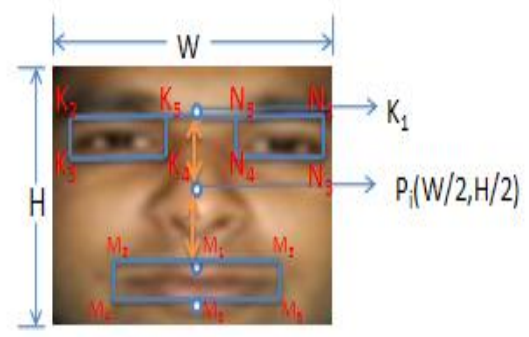

Figure 3.4 Face crop

- $\quad$ Dist $\left|\mathrm{P}_{\mathrm{i}}-\mathrm{K}_{1}\right|=\mathrm{H} / 8$

- Dist $\left|\mathrm{K}_{1}-\mathrm{K}_{2}\right|=$ Dist $\left|\mathrm{M}_{1}-\mathrm{M}_{2}\right|=$ Dist $\left|\mathrm{M}_{1}-\mathrm{M}_{3}\right|=$ Dist $\left|M_{4}-M_{5}\right|=$ Dist $\left|M_{5}-M_{6}\right|=W / 3$

- Dist $\left|\mathrm{K}_{2}-\mathrm{K}_{3}\right|=$ Dist $\left|\mathrm{K}_{4}-\mathrm{K}_{5}\right|=$ Dist $\left|\mathrm{N}_{2}-\mathrm{N}_{3}\right|=$ Dist $\left|N_{4}-N_{5}\right|=$ Dist $\left|M_{2}-M_{4}\right|=$ Dist $\left|M_{1}-M_{5}\right|=$ Dist $\mid$ $\mathrm{M}_{3}-\mathrm{M}_{6} \mathrm{I}=\mathrm{H} / 10$

- Dist $\left|\mathrm{K}_{3}-\mathrm{K}_{4}\right|=$ Dist $\left|\mathrm{K}_{5}-\mathrm{K}_{2}\right|=$ Dist $\left|\mathrm{N}_{3}-\mathrm{N}_{4}\right|=$ Dist $\left|\mathrm{N}_{5}-\mathrm{N}_{2}\right|=\mathrm{W} / 4$ 


\subsection{Features Location}

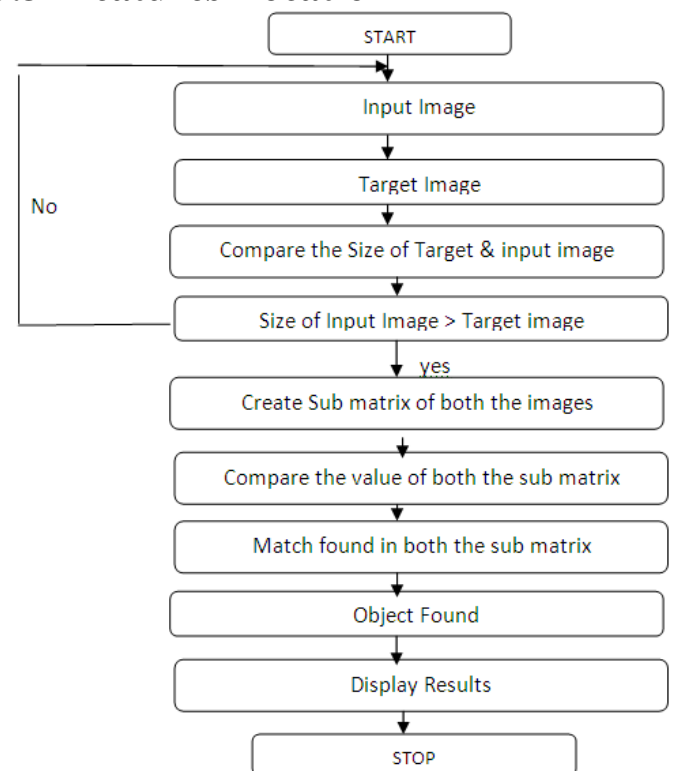

Figure 3.7 Data Flow diagram for locating features of training database into input image face region

In this venture we make a framework that is valuable to discover the protest that is available inside the picture. In this we initially take a picture as a contribution for which we need to discover the question. Subsequent to choosing the info picture we need to concentrate on the objective picture. In the wake of getting both information picture and target picture we look at the measure of both the picture. On the off chance that the measure of information picture is more noteworthy than target picture then we continue with our framework else we will concentrate on the info picture. On the off chance that the above criteria get fulfilled then we make the sub lattices of both the pictures for instance we make $3 \times 3$ frameworks. In the wake of making the sub networks we analyze both the sub frameworks of both the picture. This Matrix can be made with the assistance of pixels show in both the pictures. On the off chance that coordinating discovered then we infers that the given question is found in the other picture.

The protest is appeared as a yield by making red boxes on that question. The yield question picture is shown and the co ordinates of that protest that is the stature and width from top and base as directions is appeared with the assistance of im device.

\subsection{Fuzzy Rules \& Conclusion}

We forms too many fuzzy rules that gives us appropriate result.

Table 3.1 Fuzzy Rules Experiment Results

\begin{tabular}{|l|l|l|l|}
\hline $\begin{array}{l}\text { S. } \\
\text { No }\end{array}$ & $\begin{array}{l}\text { Images } \\
\text { tested }\end{array}$ & $\begin{array}{l}\text { Gender } \\
\text { Predicted }\end{array}$ & $\begin{array}{l}\text { \% of Gender } \\
\text { prediction }\end{array}$ \\
\hline 1 & 5 & 5 & 100 \\
\hline 2 & 10 & 7 & 70 \\
\hline 3 & 15 & 12 & 80 \\
\hline 4 & 20 & 16 & 80 \\
\hline
\end{tabular}

Male $\&$ Female Features for gender recognization $=50 \mathrm{M} / 50 \mathrm{~F}$

\section{CONCLUSION}

This paper introduced a way to deal with sex arrangement from still picture. We find that the proposed procedure framework performs by and large equivalently to Human audience members, in spite of the fact that the execution of our classifiers is more awful on short expressions. A straightforward "lion's share voting" blend ponder did not enhance order exactness, apparently because of the precise idea of disarrays. The consequences of a client think about [16] demonstrates that utilization examples of Proposed frameworks for senior residents vary altogether from those of grown-ups or youthful guests. Given that most disarray show up between neighboring classes, we trust the general acknowledgment of proposed frameworks can be expanded essentially by giving customized adaptations of such frameworks, which adjust attributes, for example, the level of robotization in a guest pre-choice situation, request of introduction of alternatives. In these situations, sex grouping is not used to restrain get to (e.g. as in security of minors), yet to build client fulfillment by giving individualized administrations even without information about the guest's character.

\section{REFERENCES}

[1] Edward D. Mysak, "Pitch duration characteristics of older males," Journal of Speech and Hearing Research, vol. 2, pp.46-54, 1959.

[2] Sue E. Linville, Vocal Aging, Singular Publishing Group, SanDiego, CA; USA, 2001.

[3] Christian M"uller, Frank Wittig, and J"org Baus, "Exploiting speech for recognizing elderly users to respond to their special needs," in Proc. Euro speech 2003, Geneva; Switzerland, Sept.2003, ISCA.

[4] Nobuaki Minematsu, Mariko Sekiguchi, and Keikichi Hirose,"Automatic estimation of one's age with his/ her speech based upon acoustic modeling techniques of speakers," in Proc.ICASSP 2002, Orlando, FL; USA, May 2002, IEEE.

[5] Izhak Shafran, Michael Riley, and Mehryar Mohri, "Voice signatures,"in Proc. ASRU 2003, U.S. Virgin Islands, Dec. 2003, IEEE.

[6] Susanne Sch"otz, "Automatic prediction of speaker age using CART," Term paper for course in Forensic Phonetics, G“oteborg University

[7] European Language Resources Association (ELRA),"http://www.speechdat.org/," http://www.elra.info/.

[8] Jitendra Ajmera, "Effect of age and gender on LP smoothed spectral envelope," in Proc. Speaker Odyssey. 2006, IEEE.

[9] Loredana Cerrato, Mauro Falcone, and Andrea Paoloni, "Subjective age estimation of telephonic voices," Speech Communication, vol. 31, no. 2-3, pp. 107-102, 2000.

[10] The FG-NET Aging Database http://www.fgnet.rsunit.com/.

[11] Y. Fu, Y. Xu, and T. S. Huang. Estimating human age by manifold analysis of face pictures and regression on aging features. Proceedings of the IEEE Multimedia and Expo, pages 1383-1386, 2007.

[12] G. Guo, G. Mu, Y. Fu, C. Dyer, and T. Huang. A study on automatic age estimation using a large database. International Conference on Computer Vision in Kyoto (ICCV 2009), pages 1986-1991, 2009. 
[13] A. E. Hoerl and R. W. Kennard. Ridge regression: Biased estimation for no orthogonal problems. Techno metrics, 12(3):55-67, 1970.

[14] T. Kanamori, S. Hido, and M. Sugiyama. A least-squares approach to direct importance estimation. Journal of Machine Learning Research, 10:1391-1445, 2009.

[15] K. J. Ricanek and T. Tesafaye. Morph: A longitudinal image database of normal adult age-progression. Proceedings of the IEEE 7th International Conference on Automatic Face and Gesture Recognition (FGR 2006), pages 341-345, 2006.

[16] B. Sch"olkopf and A. J. Smola. Learning with Kernels, MIT Press, Cambridge, MA, USA, 2002.

[17] H. Shimodaira. Improving predictive inference under covariate shift by weighting the log-likelihood function. Journal of Statistical Planning and Inference, 90(2):227244, 2000.

[18] M. Stone. Cross-valedictory choice and assessment of statistical predictions, Journal of the Royal Statistical Society, Series B, 36:111-147, 1974.

[19] M. Sugiyama, M. Kawanabe, P. L. Chui. Dimensionality reduction for density ratio estimation in highdimensional spaces. Neural Networks, 23(1):44-59, 2010

[20] M. Sugiyama, M. Krauledat, and K.-R. M"uller. Covariate shift adaptation by importance weighted cross validation. Journal of Machine Learning Research, 8:985-1005,May 2007.

[21] M. Sugiyama, M. Yamada, P. von B"unau, T. Suzuki, T. Kanamori, and M. Kawanabe.Direct density-ratio estimation with dimensionality reduction via leastsquares hetero-distributional subspace search, Neural Networks.

[22] F. H. C. Tivive and A. Bouzerdoum. A gender recognition system using shunting inhibitory convolutional neural networks. Proceedings of the International Joint Conference on Neural Networks (IJCNN '06), pages 5336-5341, 2006.

[23] K. Ueki, M. Sugiyama, Y. Ihara. A semi-supervised approach to perceived age prediction from face images. IEICE Transactions on Information and Systems, to appear

[24] K. Ueki, M. Miya, T. Ogawa, T. Kobayashi. Class distance weighted locality preserving projection for automatic age estimation. Proceedings of IEEE International Conference on Biometrics: Theory, Applications and Systems (BTAS 2008), pages 1-5, 2008.

[25] M. Yamada, M. Sugiyama, G. Wichern, and J. Simm. Direct importance estimation with a mixture of probabilistic principal component analyzers. IEICE Transactions on Information and Systems, E93-D(10), 2846-2849, 2010. 